\title{
Antifungal Property of Piper betle Leaf Oil against Oral Candida Species
}

\author{
Rattiporn Kaypetch $^{1}$ and Sroisiri Thaweboon ${ }^{2 a}$ \\ ${ }^{1}$ Research Office, Faculty of Dentistry, Mahidol University, Bangkok, Thailand \\ ${ }^{2}$ Department of Oral Microbiology, Faculty of Dentistry, Mahidol University, Bangkok, Thailand
}

\begin{abstract}
Fungal infection is one of the main clinical problems due to the extensive uses of broad-spectrum antibiotics and immunosuppressive therapy. Among all, candida species are the most prevalent. Piper betle Linn., a tropical plant intimately associated with pepper, has been widely used as a traditional herb in many Asian countries. The purpose of this study was to evaluate the antimicrobial effect of essential oil extracted from fresh leaves of $P$. betle against four strains of candida species, C. albicans, C. glabrata, C. krusei, C. parapsilosis, C. tropicalis, C. pseudotropicalis and C. stellatoidia. Inhibitory activity was primarily screened by Kirby-Bauer disc diffusion technique and subsequently the minimum inhibitory concentration (MIC) was determined by agar dilution technique. Betel oil exhibited a high potential of antifungal property against all strains of yeast with inhibition zones ranged from 32 to $33 \mathrm{~mm}$. in diameter and MIC values of $0.039-0.078 \% \mathrm{v} / \mathrm{v}$. Data from this study demonstrates a potential application of betel oil in drug preparations and development for the treatment of candida infection. Further investigations are required to define the antifungal mechanism of this oil as well as clinical trial in the patients.
\end{abstract}

\section{Introduction}

Recently, there has been a rising interest in opportunistic infections caused by pathogenic fungi. The growing concern is due to an increasing population with HIV infection and the more extensive uses of broad-spectrum antibiotics, immunosuppressive therapy and invasive surgical procedures. Of the fungi regarded as the cause of infection, candida species are the most commonly found. The genus candida comprises of a heterogeneous group of yeasts with at least 50 different species. Nonetheless, approximately $70 \%$ of invasive infections are caused by Candida albicans, Candida glabrata, Candida, parapsilosis, Candida tropicalis, C. pseudotropicalis and Candida krusei [1]. The virulence of these yeasts is ascribed to the capacity to avoid host defenses, adherence and biofilm formation on host tissue, and the releasing of hydrolytic enzymes that can damage tissues [2].

In dentistry, candida infection in the oral cavity, oral candidiasis, can present in many forms according to how deeply the organism has spread, or if host defenses allow for more consequential infections. The most common form of candida infection is oral thrush which is also known as pseudomembranous candidiasis. This form of mucosal infection is usually described by a white cottage cheese-like plaque or biofilm that can be wiped off to disclose a red bleeding base. The plaque that can be removed is generally composed of aggregates of the candida hyphae and by products of epithelial breakdown. This infection is seen in high risk people such as infants and young children, whose host defenses have yet to

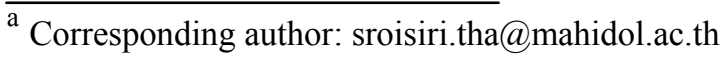

develop, or in the elderly with a decline in immune defenses.

In addition to infants and aging people, oral candidiasis has increased due to the spread of HIV infection. In this regards, oropharyngeal candidiasis is a clinical indicator of HIV disease advancement.

Few antifungal drugs are accessible compared with the wide range of antibiotics for bacterial infections. This may indicate the difficulty implicated in developing an agent with property against a eukaryotic cell type without host toxicity. Some commercially available antifungal agents are brought to use for the treatment of candida infection, however, these drugs had been reported to produce adverse effects such as allergic reactions, interaction with other medicines, and drug resistance.

In order to solve the unfavourable effects of commercial products and to control candida infection, the development of natural products capable of clinical application is required. Natural products have been evidenced to be an alternative to synthetic chemical agents and the remark in medicinal herbs as a source of antimicrobial agents has increased rapidly. Piper betle Linn. or betel is a tropical plant intimately associated with pepper which is extensively grown in many Southeast Asian countries such as Thailand, Malaysia and India. It is a root climber with deep green heart shaped leaves. Betel leaves are the most important part and are of medicinal, religious and ceremonial value. Chewing betel leaves is supposed to prevent bad breath, harden the gum, conserves the teeth and sweetens breath. The infusion prepared from the leaves has been known as traditional herbs used as mouthwash, cough medicine, 
tonic or astringent [3]. Recently, oil from betel leaves has been concluded to be a promising agent in controlled and released food packaging technology by its antimicrobial activity against food spoilage yeasts [4].

The purpose of this study was to evaluate the antifungal effect of essential oil extracted from fresh leaves of $P$. betle against seven strains of candida species.

\section{Materials and Methods}

\subsection{Oil}

Betel oil was extracted from fresh leaves of $P$. betle by hydrositillation by the Thailand Institute of Scientific and Technological Research (TISTR) and prepared in 5\% dimethyl sulfoxide (DMSO).

\subsection{Candida species}

The yeast strains used in the study were Candida albicans ATCC 10231, Candida krusei ATCC 6258, Candida glabrata ATCC 90030, Candida parapsilosis ATCC 22019, Candida tropicalis (clinical isolate), Candida pseudotropicalis (clinical isolate) and Candida stellatoidia (clinical isolate). All of them were obtained from a culture collection of Oral Microbiology Department, Faculty of Dentistry, Mahidol University, Thailand. Sabouraud dextrose broth (Difco, USA) was used to culture all yeast strains and the suspension of $10^{7}$ $\mathrm{CFU} / \mathrm{ml}$ of yeast (equivalent to $0.5 \mathrm{McFarland}$ Standard) was used for antimicrobial evaluation.

\subsection{Disc diffusion test}

The inhibitory potential of betel oil on fungal growth was evaluated using the disc diffusion technique described by Kirby-Bauer [5]. In brief, freshly prepared yeast suspension was spread on Mueller Hinton agar (Difco, USA). A $20 \mu \mathrm{L}$ of betel oil was dropped onto sterile paper disc (6 mm. in diameter) and left for $60 \mathrm{sec}$. Then, paper disc soaked with oil was put on the agar surface and the plate was incubated at $37^{\circ} \mathrm{C}$ for $24-48 \mathrm{~h}$. The same volume of $0.2 \%$ chlorhexidine gluconate solution and 5\% DMSO solution served as positive and negative controls, respectively. After incubation, the zones of inhibition surrounding paper disc were measured using a Vernier caliper.

\subsection{Minimum inhibitory concentration (MIC)}

The MICs of betel oil on candida were investigated with the agar dilution technique. Mueller Hinton agar plates were prepared with serial two-fold dilutions of betel oil (ranged from 20 to $0.02 \% \mathrm{v} / \mathrm{v}$ ). The yeast suspensions were prepared by adjusting their density to 0.5 McFarland Standard. The $20 \mu \mathrm{L}$ of suspension were dropped on each plate and incubated at $37^{\circ} \mathrm{C}$ for $48 \mathrm{~h}$.

All tests were carried out in triplicate on three consecutive times.

\section{Result}

By using disc diffusion method, betel oil exhibited inhibitory effect on the growth of candida species (Table 1). The minimum inhibitory concentration (MIC) ranged from 0.039 to $0.078 \% \mathrm{v} / \mathrm{v}$ (Table 2). According to higher MIC value, C. albicans, C. krusei and C. tropicalis were more resistant to the oil.

Table 1. Inhibitory effect of betel oil against various candida species

\begin{tabular}{|c|c|c|c|}
\hline \multirow{2}{*}{ Candida species } & \multicolumn{3}{|c|}{ Inhibition zone (mm) } \\
\hline & oil & + ve control ${ }^{a}$ & -ve control ${ }^{b}$ \\
\hline C. albicans & $33.83 \pm 0.76$ & $22.83 \pm 0.28$ & - \\
\hline C. glabrata & $33.83+0.76$ & $23.50 \pm 0.50$ & - \\
\hline C. krusei & $32.66 \pm 0.57$ & $23.33+0.57$ & - \\
\hline C. parapsilosis & $33.83+0.76$ & $24.33+0.57$ & - \\
\hline C. tropicalis & $30.83+0.28$ & $23.83+0.28$ & - \\
\hline C. pseudotropicalis & $33.50 \pm 0.50$ & $24.16+0.28$ & - \\
\hline C. stellatoidia & $35.50 \pm 0.86$ & $25.83 \pm 0.28$ & - \\
\hline
\end{tabular}

${ }^{a}$ positive control was $0.2 \%$ chlorhexidine gluconate

${ }^{\mathrm{b}}$ negative control was 5\% DMSO values are expressed as mean $\pm \mathrm{SD}$

Table 2. Minimum inhibitory concentration (MIC) values of betel oil against various candida species.

\begin{tabular}{|l|c|}
\hline Candida species & MIC $(\mathbf{\%} \mathbf{v} / \mathbf{v})$ \\
\hline & \\
C. albicans & 0.078 \\
\hline C. glabrata & 0.039 \\
\hline C. krusei & 0.078 \\
\hline C. parapsilosis & 0.039 \\
\hline C. tropicalis & 0.078 \\
\hline C. pseudotropicalis & 0.039 \\
\hline C. stellatoidia & 0.039 \\
\hline
\end{tabular}

\section{Discussion}

Regarding the rising occurrence of drug resistant pathogenic fungi and the toxicity of current antifungal drugs, the search for natural products that exhibit 
antimicrobial properties as an alternative treatment has been suggested. Plants have been used in medicine for a long time since they represent an economic alternative, are easily obtainable and can be applied to several pathologies [6]. Piper betle leaves have been traditionally used in folk medicine for the cure of various disorders and for chewing purposes together with other seasoning. The ethnic groups in many Asian countries such as Sri Lanka, India, and Thailand, chew the leaves as a narcotic which induces swoon and excessive sweating and also helps to give warmth to the body during the cool season. Betel oil has been shown to possess antimicrobial, gastroprotective, hepatoprotective, wound healing and antioxidant activities [7].

The results from the present study demonstrated antifungal properties of betel oil against all tested strains of candida with a zone of inhibition ranged from 32 to 35 $\mathrm{mm}$. in diameter compared with 22 to $25 \mathrm{~mm}$. of chlorhexidine solution, a positive control. The MIC values of the oil against candida were $0.039-0.078 \% \mathrm{v} / \mathrm{v}$. In this regards, C. albicans, C. krusei and C. tropicalis exhibited more resistant to the oil. Previous studies have been conducted using essential oil and ethanolic extract of $P$. betle to evaluate antimicrobial activity against bacteria and fungi $[3,7]$. The growth inhibitory effect was reported against various gram positive bacteria (Enterococcus faecalis, Streptococcus pyogenes, Bacillus cereus, Micrococcus luteus), gram negative bacteria (Escherichia coli, Salmonella enteritidis) and yeasts (Candida albicans, Saccharomyces cerevisiae). In the field of dentistry, anti-halitosis activity of betel leaf extract was demonstrated due to the reduction of methyl mercaptan and hydrogen sulfide produced by obligate oral anaerobes (Porphyromonas gingivalis and Fusobacterium nucleatum) [8]. Furthermore, it has also been reported that aqueous extract could inhibit growth, adherence and extracellular polysaccharide formation of caries-associated bacteria (Streptococcus mutans, Streptococcus mitis and Actinomyces spp.) suggesting its potential role in controlling dental plaque development $[9,10]$.

$P$. betle contains a vast variety of biologically active components whose concentration bases on the diversity of the plant, climate, harvesting period and geographic location. The major components of betel oil were reported to be safrole, chavibetol acetate, allylpyrocatechol diacetate, methyl chavicol, methyl eugenol, 1,8-cineole, eugenol, caryophyllene, carvacrol, pinene, limonene, campene and cadinene $[7,11]$. However, the most active antimicrobial components appear to be carvacrol, eugenol and chavibetol [12]. The antifungal effects were attributed to their ability to inhibit ergosterol biosynthesis of yeast cell and to make the cytoplasmic membrane porous [13]. These result in the disruption of cytoplasmic membrane integrity. In addition, active component such as carvacrol has a hydroxyl group on the phenolic ring of its structure which increases hydrophilic ability and help it dissolve in the microbial membranes and impair them. Previous study [14] proposed mechanism of action of $P$. betle extract on candida that it involved the aberration of cell wall elements and the damage of cytoplasmic membrane, causing coagulation of cell components and the efflux of critical molecules and ions which lead to an autolytic phase that finally brings about cell death.

In the case of safety profile, studies in animal models revealed that betel oil was safe in terms of hepatotoxicity, renotoxicity, hematotoxicity, gross morphology, stress or aversive behaviours $[15,16]$. Oral administration of betel extract showed protection against gastric damage induced by absolute ethanol by the increase of mucus content [17]. Furthermore, Nilugal et al [18] reported that ointment containing betel extract could enhance the rate of wound contraction and reduce the healing period when applied to rats.

In conclusion, data from this in vitro study demonstrate that essential oil from $P$. betle leaves exhibits inhibitory effect on candida spp. It might have a potential application in drug preparations and development for the treatment of candida infection. However, further investigations are required to define the antifungal mechanism of this oil as well as clinical trial in the patients.

\section{Acknowledgement}

The Authors would like to thank the Thailand Institute of Scientific and Technological Research (TISTR) for providing us with Piper betle oil, along with Associate Prof. Boonyanit Thaweboon for his valuable advice on various technical issues, and Miss Thaniya Muadcheingka for her kind assistance in preparing culture media.

\section{References}

1. M.A. Pfaller, Diekema, Clin. Microbiol. Rev. 20, 133 (2007)

2. S. Silva, M. Negri, M. Henriques, R. Oliveira, D.W. Williams, J. Azeredo, F.E.M.S. Microbiol. Rev. 36, 288 (2012)

3. M.P. Rai, K.R. Thilakchand, P.L. Palatty, P. Rao, S. Rao, H.P. Bhat, M.S. Baliga, Asain. Pacific. J. Cancer. Prev. 12, 2149 (2011)

4. P. Suppakul, N. Sala-Ead, P. Phoopuritham, Kasetsart. J. 40, 91 (2006)

5. A.W. Bauer, W.M. Kirby, J.C. Sherris, Am. J. Clin. Pathol. 36, 493 (1966)

6. M.C. Cruz, P.O. Santos, A.M. Jr Barbosa, D.L. de Mélo, C.S. Alviano, A.R. Antoniolli, D.S. Alviano, R.C. Trindade, J. Ethnopharmacol. 111, 409 (2007)

7. L.S. Arambewela, L.D. Arawwawala, K.G. Kumaratungan, D.S. Dissanayake, W.D. Ratnasooriya, S.P. Kumarasingha, Pharmacogn. Rev. 5, 159 (2011)

8. N. Ramji, R. Iyer, S. Chandrasekaran, J. Ethnopharmacol. 83, 149 (2002)

9. Z.H. Rahim, N. Thurairajah, J. Appl. Oral. Sci. 19, 37 (2011)

10. F.A. Razak, Z.H. Rahim, J. Oral. Sci. 45, 201 (2003)

11. A.M. Rimando, Arch. Pharm. Res. 9, 93 (1986)

12. H.J. Dorman, S.G. Deans, J. Appl. Microbiol. 88, 
$308(2000)$

13. A. Ahmad, A. Khan, F. Akhtar, S. Yousuf, I. Xess, L.A. Khan, N. Manzoor, Eur. J. Clin. Microbiol. Infect. Dis. 30, 41 (2011)

14. M.A. Nordin, W.H. Wan Harun, F. Abdul Razak, M.Y. Musa, Int. J. Oral. Sci. 6, 15 (2014)

15. L.S. Arambewela, W.D. Ratnasooriya, L.D. Arawwawala, Trop. Med. Plants. 4, 195 (2003)

16. A. Sengupta, P. Adhikary, B.K. Basak, K. Chakrabarti, P. Gangopadhyay, J. Banerji, A. Chatterjee, Indian. J. Exp. Biol. 38, 338 (2000)

17. L.D. Arawwawala, L.S. Arambewela, W. Ratnasooriya, J. Ayurveda. Integr. Med. 5, 38 (2014)

18. K.C. Nilugal, K. Perumal, R.E. Ugander, Am. J. Pharm. Tech. Res. 4, 443 (2014) 